\title{
La salud: una esperanza para los tiempos de crisis
}

\author{
José Manuel Ribera-Casado
}

Bienvenidos a esta sala, a esta facultad y a esta universidad. Quiero brindar a todos ustedes una bienvenida personal, pero, sobre todo, institucional. Lo hago en nombre del Ilmo. Sr. Decano, Prof. José Luis Álvarez Sala, de toda la facultad, de quienes van a ser sus profesores, de los que sin serlo van a contribuir a su formación desde otros cometidos, administrativos, personas que trabajan en este centro en otras funciones, e incluso en el de sus compañeros de cursos superiores. Una bienvenida personalizada dirigida a cada uno de quienes inician hoy los estudios de medicina, de nutrición o de terapia ocupacional. Bienvenidos al lugar donde van a pasar la mayor parte de su tiempo durante los próximos años.

Hoy es su primer día académico. Durante treinta minutos pretendo compartir reflexiones y transmitir algunos mensajes que puedan servir de guía para el camino que hoy se abre para todos ustedes. Unos mensajes que intentan ser optimistas y cargados de esperanza. El optimismo y la esperanza son las mejores armas para afrontar estos tiempos de crisis en la que según parece estamos todos inmersos.

\section{Una buena elección}

Empezaré por anunciar que, a mi juicio, ustedes han hecho una buena elección. Por eso mi primera reflexión y también mi primer mensaje tienen que ver con la naturaleza de la profesión elegida. Muchos compañeros suyos se han incorporado hoy en ésta y en otras universidades a diferentes facultades. Van a ser expertos en temas muy importantes, todos de altísimo interés, y lo hacen con la misma ilusión que tienen ustedes. Serán abogados que lucharán por una sociedad más justa, ingenieros o arquitectos que buscarán hacer nuestra vida más cómoda y confortable, biólogos, químicos, filósofos, físicos, profesionales de numerosas ramas del saber que, desde diferentes perspectivas, van a intentar contribuir al bien común, al avance de las ciencias y al de todo tipo de conocimientos. En definitiva, contribuir al progreso y hacer este mundo nuestro más habitable.

Ustedes van por delante. Han elegido lo mejor. Serán profesionales de la salud. Los encargados de prevenirla, conservarla y recuperarla. Y la salud es lo más valioso que tiene el hombre. Así lo sentimos todos, no sólo los que nos dedicamos a este oficio, sino el conjunto de la sociedad. Lo dicen las encuestas donde quiera que se lleven a cabo. Y lo dicen de una manera tanto más contundente cuanta mayor es la experiencia de quien habla, cuanto más elevada es la edad de las personas a las que se pregunta.

Inician hoy una nueva etapa de su vida y lo hacen habiendo optado por una profesión bella y eterna. Una profesión atractiva por sus contenidos y por la forma de ejercerla, siempre próxima a otros hombres y mujeres que van a poner en ustedes su confianza, sus esperanzas, sus afectos y, también, muy importante, su agradecimiento. Una profesión solidaria, al servicio de los demás. Al servicio, sobre todo, de aquellas personas y colectivos más necesitados. Ustedes van a dar salud y salud es sinónimo de felicidad. Serán los encargados de generar esa felicidad.

Siempre ha habido personas dedicadas a proporcionar salud a sus semejantes, médicos en un sentido genérico. Pero cada vez más, al médico en sentido estricto, se le han ido sumando en su trabajo diario profesionales de otras áreas, cuidadores en el sentido más amplio de la expresión, siempre buscando opciones más positivas para mantener y mejorar la salud. Todos caminamos en la misma dirección. Por ello, cuando hoy hablo de medicina y de médicos, deben entender que me dirijo a todos y cada uno de ustedes con independencia de la ventanilla en la que se hayan inscrito. Nuestro fin es el mismo, aunque los caminos puedan ser distintos.
Catedrático Emérito de Geriatría. Facultad de Medicina. Universidad Complutense de Madrid. Madrid, España.

E-mail: jribera.hcsc@salud.madrid.org Nota: Este artículo constituye el discurso de inauguración del curso 2012-2013 de la Facultad de Medicina de la Universidad Complutense de Madrid.

Conflicto de intereses: No declarado.

Competing interests: None declared.

(c) 2013 FEM 
El hecho de reunir en este acto a estudiantes que han optado por distintas ramas de lo que se conoce como ciencias de la salud no hace sino corroborar una de las características de la medicina moderna, la necesidad de trabajar en equipo. Oirán hablar en el curso de su carrera de equipos multi e interdisciplinares, donde actuamos de la mano las diferentes profesiones sanitarias. Incluso se incorporan trabajadores procedentes de campos cuya dedicación a las cuestiones relacionadas con la salud puede ser meramente tangencial. Todos sumamos. Todos participamos de un trabajo en equipo que contempla al hombre enfermo, al paciente, en su totalidad. Que suma conocimientos y experiencias en la búsqueda de soluciones globales. Hacerlo así representa la forma más eficaz de conseguir aquello que da razón de ser a la profesión. Por ello, aunque las palabras 'medicina' y 'médico' sigan siendo representativas, aporten una carga simbólica y afectiva enorme, y yo las utilice repetidamente en este acto, cada vez en mayor medida escucharán otras expresiones alternativas como la de 'ciencias de la salud' o la de 'profesiones sanitarias'. Todos entramos en el mismo saco aunque los enfoques puedan ser distintos en cada caso.

La medicina siempre ha sido una profesión respetada y prestigiada. Lo sigue siendo. Una encuesta reciente en nuestro país valora con un 8,5 sobre 10 la contribución de la profesión médica al bienestar y al avance de la sociedad. Representa la opción más valorada entre los doce colectivos profesionales sobre los que se pregunta [1]. Se trata de una profesión tremendamente atractiva para el conjunto de la sociedad, tanto por los fines que persigue como por las formas que utiliza para alcanzarlos.

He aludido ya a sus fines, situando la salud como referente fundamental, como el telón de fondo desde donde toma perspectiva todo nuestro trabajo. Mantener la salud implica en primer término prevención, impedir que esta salud falle por cualquier motivo. El concepto de medicina preventiva es relativamente reciente, corresponde al siglo $\mathrm{xx}$, sobre todo a su segunda mitad, y ha crecido en paralelo a como lo ha venido haciendo el conocimiento de lo que hace aproximadamente sesenta años se bautizó con el nombre de 'factores de riesgo'. La importancia de la medicina preventiva la podemos calibrar si tenemos en cuenta que es la principal responsable de que la esperanza de vida al nacer se haya multiplicado por dos y medio durante el siglo xx. Un español nacido en 1900 tenía una esperanza de vida media de 35 años, lo que implica que solamente el 50\% de los nacidos en aquel momento iba a alcanzar esa edad. Un siglo después, en el año 2000, la esperanza de vida media al nacer de nuestros compatriotas estaba en torno a los 80 años. Y sigue subiendo.

Pero, evidentemente, los objetivos de la medicina no se circunscriben a la prevención. Implican también la atención directa al enfermo, algo que está en la propia raíz de la profesión y que ha sido la tarjeta de presentación del médico a lo largo de los siglos. En esta tarea de la atención inmediata, los pasos sucesivos desde un punto de vista conceptual son la capacidad para reconocer la enfermedad (el diagnóstico) y la aplicación prudente en cada situación de los procedimientos más adecuados frente a ella (tratamientos y procedimientos rehabilitadores). Implica hacer investigación, tarea ingente siempre inacabada para la cual, habitualmente, el equipo -de nuevo el equipo- va a integrar a profesionales procedentes de otros campos. No hay tiempo para comentar estos puntos. Irán escuchando estos conceptos y aplicando lo que de ellos se desprende a lo largo de la carrera y, más tarde, en su ejercicio profesional.

Decía que el respeto de la sociedad en su conjunto hacia nuestra profesión viene tanto por los fines como por la forma de ejercerla, por el método. Sobre este tema, la relación entre el médico y el enfermo como forma de ejercer la medicina, existe una bibliografía muy numerosa en todas las lenguas y a lo largo de la historia. Para ceñirme únicamente a épocas recientes y a nuestro propio medio, mencionaré a dos ilustres profesores de esta facultad que han sido referentes en este campo durante la primera y la segunda mitad del siglo xx: Gregorio Marañón y, más tarde, Pedro Laín Entralgo. Sus escritos en este sentido, como los más recientes del también profesor de esta facultad Diego Gracia Guillén, son, a mi juicio, lecturas obligadas para cualquier estudiante que quiera profundizar en la esencia de la profesión.

Entre los muchos elementos básicos sobre los que se sustenta este ejercicio profesional destacaría la proximidad 'al otro', tal como lo definía en uno de sus libros el Prof. Laín Entralgo. La búsqueda de su empatía, el respeto a sus confidencias, la generosidad propia a la hora de dedicarle tiempo y atención. En general, la priorización en la forma de actuar de todo eso que llamamos 'valores', por encima de cualquier otra consideración centrada en aspectos puramente técnicos, por más que estos deban ser siempre bien conocidos y aplicados de forma correcta por parte del profesional.

Uno de los elementos novedosos en la manera de entender hoy la medicina en relación a lo que se consideraba doctrina sólida hace apenas unas décadas estriba en la evolución de esta relación médico- 
enfermo. Se ha pasado del denominado 'paternalismo benevolente', heredero de la tradición hipocrática, a una relación mucho más horizontal y acorde con los tiempos. Una relación en la que el paciente asume mayor protagonismo, que llega a ser decisivo a la hora de la toma de decisiones. Se habla de una 'medicina centrada en el paciente' en contraposición a la 'medicina centrada en el médico' [2]. En la raíz de este cambio se encuentra el hecho de que, durante el último tercio del pasado siglo, las minorías de todo tipo han buscado y en gran parte han conseguido hacer valer lo que genéricamente se han considerando 'sus derechos'. En este contexto hay que entender el cambio en la relación médico-enfermo. Un cambio que, además, no se ha limitado al campo de la actividad clínica, sino que se ha introducido también en la esfera de la investigación biomédica y, en general, en la manera de relacionarse la actividad médica con el conjunto de la sociedad [3].

Todo ello constituye la grandeza de nuestra profesión y justifica mi afirmación de que han elegido bien.

\section{Algunas características de la profesión}

¿Con qué se van a encontrar? Vienen ustedes a la facultad a adquirir información y formación. Información y conocimientos acerca de lo que la medicina es hoy y acerca de cómo ejercerla. Formación para integrar esos conocimientos y saber aplicarlos en la práctica médica a partir del primer encuentro con un enfermo. Por ello, éste podría ser un momento adecuado para hablar de cuestiones prácticas muy concretas relativas al hoy: exponer cómo está organizada la facultad, comentar las características de los planes de estudio, describir los grandes apartados en los que se divide la enseñanza médica pregraduada -periodo básico o preclínico y periodo clínico- o de sus equivalentes en los grados de nutrición y de terapia ocupacional. Hablar también de otras cuestiones que, sin duda, tienen un enorme interés y han estado rondando por su cabeza durante estos meses: las prácticas, los exámenes, las ventajas o inconvenientes de los diferentes hospitales por los que tendrán oportunidad de pasar, las inquietudes que según avanza la carrera genera la sombra del examen MIR, las eventuales salidas profesionales, etc.

Podría hablar del denominado 'Plan Bolonia, donde se explicita la necesidad de adquirir conocimientos (saberes), pero también habilidades y actitudes. Los saberes y las habilidades los tendrán que aprender durante la carrera. También después, durante la residencia, quienes aspiran a médicos. Y todos, médicos, nutricionistas y terapeutas, deberán mejorarlos a lo largo de toda su vida profesional. La actitud, sin embargo, hay que traerla puesta. Sólo a partir de ahí se puede contribuir a su desarrollo.

No voy a entrar en ninguno de estos aspectos concretos. Son cuestiones que conocerán ustedes mismos de forma inmediata. Sólo diré que han acudido a un lugar idóneo para aprender el oficio. Un lugar reconocido oficialmente como el más adecuado para hacerse médico nada menos que desde el siglo XV. Una institución que también ha sido pionera desde hace décadas en el diseño y puesta en marcha de los programas curriculares de nutrición y de terapia ocupacional. Han elegido bien. Les aseguro que tendrán un profesorado competente y experto y que disfrutarán de una infraestructura adecuada a todos los niveles. Los resultados van a depender, sobre todo, de ustedes mismos. Su capacidad individual está contrastada a partir de una alta calificación en el examen de la selectividad. Cuánto van a aprender y hasta dónde van a llegar dependerá de lo que cada cual se aplique en el estudio, de su interés y dedicación, y de la habilidad para saber elegir lo más adecuado en cada momento en cuanto a distribución del tiempo, prioridades, etc. Debo recordar aquí que los dos únicos premios Nobel de Medicina españoles han pasado por esta facultad, donde, además, uno de ellos ejerció como profesor durante casi treinta años.

Prefiero dedicar los minutos que quedan a comentar algunas características de la profesión y a poner énfasis en determinados aspectos que constituirán su marco referencial, primero como estudiantes, pero también, más adelante, como profesionales. Comentaré aquellas que a mí me parecen más relevantes, dentro del cambio permanente que experimenta la medicina.

La medicina es una ciencia viva para cuyo ejercicio se requieren conocimientos y habilidades. Es una ciencia donde cada día aparecen evidencias nuevas que derriban conceptos y creencias que en ocasiones se han mantenido como axiomas durante décadas. Los conocimientos que yo adquirí durante la carrera no me hubieran valido hoy para aprobar una sola asignatura del currículo. Se dice que el 75\% de lo que constituye nuestro bagaje científico en un momento determinado ha quedado obsoleto apenas cinco años más tarde. Eso nos convierte en unos profesionales permanentes del estudio, mucho más allá del tiempo que podamos pasar en la facultad. Lo deberemos ser al menos hasta la jubilación. Nos compromete de por vida con lo que se conoce como 'formación continuada'. 
Este año 2012 se celebra el $200 .^{\circ}$ aniversario del nacimiento de una de las principales revistas científicas en el campo de la medicina, The New England Journal of Medicine. Las revistas médicas de primer nivel los van a acompañar a lo largo de su vida y ésta es una de las más emblemáticas. En el artículo editorial conmemorativo de este doble centenario [4], el editorialista ponía énfasis en el papel desempeñado por la publicación como crónica viva de la evolución de la biomedicina y remarcaba algunos de los logros más importantes de la medicina durante estas centurias. Destacaré uno muy relevante: la implantación de unas estrategias de inmunización que dieron como fruto principal la erradicación en todo el mundo de la viruela. El 8 de mayo de 1980, la Asamblea Mundial de la Salud de forma oficial declaraba erradicada la viruela en el mundo, como culminación de un programa iniciado en 1967, un año en el que se declararon 10 millones de casos en 43 países [5].

También se alude a otros avances de los que ha sido testigo la publicación [6]. Por ejemplo, el cambio radical que representa la posibilidad real de curar numerosas infecciones y otros procesos muy variados, incluidos muchos tumores malignos; la aparición progresiva de miles de fármacos con utilidad contrastada para toda suerte de procesos; los avances quirúrgicos, con la puesta en escena de técnicas como la cirugía mínimamente invasiva o la robótica; la secuenciación del genoma humano, etc.

Fuera ya del ámbito estrictamente clínico, los avances han sido y continúan siendo espectaculares. Citaré tres. Avances en la educación médica con formas de aprendizaje cada vez más completas, basadas en las nuevas tecnologías y modificables de acuerdo con los tiempos. En segundo lugar, la aparición y el desarrollo de las especialidades médicas y de las sociedades científicas, y en tercer lugar, la implantación de unos sistemas de salud que progresivamente alcanzan al conjunto de la población en los diferentes países. Otro cambio espectacular es el que ha representado la incorporación de la mujer. Hoy, aquí, son mayoría, pero se puede recordar que hasta 1945 no se admitieron mujeres en la Facultad de Medicina de la Universidad de Harvard y que eso se debió a la disminución de candidatos varones tras la Segunda Guerra Mundial. Diez años después todavía se limitaban las prácticas a las mujeres estudiantes y médicos 'porque debían atender sus deberes maternales' [7].

Ya he hecho referencia a dos de las características de la medicina que se van a encontrar: la puesta en valor de todo lo referido a la prevención y la necesidad progresiva de establecer equipos de colabo- ración que, de manera conjunta, permitan una mejor planificación de la atención médica, tanto en el plano individual como en el colectivo. Como complemento, me limitaré a enunciar algunas otras de estas características que a mi juicio tienen mayor relieve en la medicina actual.

Destacaré primero lo que se ha calificado como un cambio de paradigma en materia de salud. Durante siglos, la medicina se ha centrado únicamente en el paciente, el sujeto enfermo que acudía en busca de ayuda. En el último medio siglo, esta perspectiva se ha modificado y ampliado de manera que, además de la búsqueda de una mejora en la salud del individuo, se orienta complementariamente hacia la colectividad en cuanto tal. Intenta mejorar la salud de las poblaciones mediante sistemas que alcancen a la totalidad de sus individuos y que cubran las diferentes situaciones de pérdidas de salud que pudieran originarse. Además, busca lograr ambos objetivos con unos costes lo más ajustados posibles [8]. Al paradigma de la actuación individual, se han añadido el de la planificación sanitaria y el de la eficiencia, una palabra clave en estos momentos.

Una característica muy importante en la medicina actual es la que tiene que ver con el mundo de la bioética. Se trata de un concepto en alza durante los últimos cuarenta años que ha venido a superar y hacer más complejo aquello que con anterioridad daba nombre en el currículo a la asignatura de 'deontología médica'. El concepto de bioética va más allá del buen hacer del médico, incorpora en sus reflexiones otros elementos como son el propio paciente y su entorno. En su desarrollo doctrinal ha establecido unas bases sólidas propias a partir de principios como el de autonomía, el de justicia (o no discriminación), el de beneficencia y el de no maleficencia. En estos momentos, y así creo que lo deben asumir desde hoy, la bioética sobrevuela cualquier decisión médica, tanto aquellas que se plantean en el plano individual -situaciones concretas referidas a un paciente- como las que lo hacen desde una perspectiva orientada a la colectividad -aquellas relacionadas con los sistemas de salud o con la distribución de los recursos existentes-.

El respeto a sus principios fundamentales cobra especial interés en el caso de los grupos sociales más desfavorecidos o con menos capacidad de defensa: los niños, los ancianos, las personas dependientes, las minorías raciales, la población emigrante, etc. También en algunas situaciones específicas, como las que ocurren en torno al proceso de morir, las relativas a la equidad en el reparto de unos recursos económicos o tecnológicos limitados, las de- 
cisiones en las que se contraponen riesgos y beneficios en equilibrio ante alternativas médicas o quirúrgicas complejas, etc.

Otra característica de nuestro mundo con enormes consecuencias en el campo de la salud es el envejecimiento poblacional. He destacado el aumento de la esperanza de vida producido en España a lo largo del siglo xx. Algo equivalente ha tenido lugar en todos los países desarrollados y con cierto retraso en el resto del mundo. El $17 \%$ de los españoles tiene hoy más de 65 años -apenas el $5 \%$ hace un siglo-, pero el mayor grado de crecimiento relativo se produce entre los octogenarios, nonagenarios y centenarios. Si tenemos en cuenta que enfermedades muy comunes -cardiovasculares, neurodegenerativas, osteoarticulares, metabólicas o tumorales- se concentran en este segmento de población, entenderemos que el médico de mañana necesite conocer bien los cambios que tienen lugar en la fisiopatología del organismo durante el proceso de envejecer y la forma de afrontarlos tanto desde el punto de vista diagnóstico como terapéutico.

Además, en este segmento de población los problemas de salud se suelen asociar a pérdidas físicas o mentales y van casi siempre de la mano de problemas sociales. Las limitaciones funcionales para llevar a cabo algo tan simple como las actividades básicas de la vida diaria alcanzan a una tercera parte del colectivo, lo que genera dependencia y requiere un enorme esfuerzo organizativo a la hora de planificar una atención que debe contemplar de manera global lo social y lo sanitario.

En este mismo contexto del envejecimiento poblacional podemos incluir otro tema clave en la medicina de hoy, el que se deriva de la necesidad de prestar una atención especial a las enfermedades crónicas y a lo que con una palabra horrible algunos llaman 'enfermo pluripatológico'. Vivir más -la victoria de la medicina sobre numerosos procesos agudos que hace apenas unas décadas se consideraban 'mortales de necesidad' - ha conducido en muchos casos a la cronificación de las situaciones que los originaban. con la consecuente necesidad de abordar un seguimiento prolongado de estas enfermedades. Lo habitual en una persona de edad avanzada es que coincidan en ella diversos procesos crónicos. Esto implica por parte del médico, en primer lugar, la necesidad de reconocerlos, saber que existen, sacarlos a flote para poder tratarlos, pero también tomar medidas para asegurar lo que se denomina una buena 'adhesión terapéutica', es decir, el cumplimiento adecuado por parte del paciente de las normas para mantener la salud o, al menos, un buen estado funcional.
La búsqueda permanente de sistemas de salud más eficientes, pero también universales y equitativos, es otra de las características de la medicina del siglo Xxi. En 1948, el Reino Unido puso en marcha el National Health Service, un sistema de atención médica que buscaba la justicia y la universalidad y que demostró sus virtudes en muy poco tiempo. Ello hizo que sirviera de modelo para los sistemas de salud implantados después en otros muchos países, incluida España, cuya Ley General de Sanidad de 1986 asumía buena parte de sus principios. El aumento de la demanda en materia de salud, el coste creciente de las nuevas tecnologías, la búsqueda de negocio desmedido en este campo por parte de diferentes instituciones y colectivos, la propia crisis económica e, incluso, una interpretación interesada de la tan manida lucha por la eficiencia, han hecho que el riesgo de retroceso en este campo sea algo más que una amenaza.

\section{Mensajes finales}

Voy a terminar y lo haré como empecé, con una llamada a la esperanza y a la ilusión. Nos recuerdan cada día que estamos en crisis. Una crisis que nos toca a todos, pero que, no lo olviden, afecta especialmente a los miembros más vulnerables de la sociedad. Es en ese contexto donde ustedes van a ejercer una profesión bonita y eterna. Serán los profesionales de la esperanza. Durante los próximos años aprenderán doctrina y aprenderán método. Pero, junto a ello, tendrán la oportunidad de desarrollar unos valores básicos y universales tanto para el ejercicio de la profesión como para la vida en su conjunto. El valor de la proximidad, del diálogo, de la cercanía, del compartir. Todo ello está en la esencia de la medicina y poder vivirlo desde dentro los convierte en unos privilegiados dentro del conjunto de la sociedad.

Durante su paso por la facultad se van a convertir en expertos en diferentes cuestiones concernientes a la salud. Pero, al mismo tiempo, en expertos en la comunicación, capaces de poner en valor cada día lo que representa la palabra. Van a ser los que dan la mano, como decía un ilustre médico gallego, el Dr. Domingo García Sabell. Con crisis o sin crisis ello los convierte, por encima de todo, en los encargados de transmitir felicidad. La salud es felicidad por encima de cualquier otra cosa. Así lo entiende la gente de la calle, las personas con las que van a convivir cada día, y así lo expresan cada 22 de diciembre mientras rompen los boletos de esa lotería que nunca toca. 
Deberán desarrollar su actividad, como estudiantes primero y como profesionales después, con entusiasmo y con optimismo. Con la conciencia de quien sabe que está en deuda con la sociedad, que debe responder al privilegio de haber podido prepararse para ejercer esta profesión. Deberán asumir la responsabilidad de haberse convertido en lo que los expertos llaman 'líderes de opinión'. Lo serán por encima de cualquier amenaza que puedan encontrar. Entre ellas, una de las más peligrosas es la inercia, el dejarse ir. Hay que prepararse desde ahora para superarla. Estar dispuesto a renovarse, actualizarse cada día, es la mejor manera de hacerlo. El presidente Kennedy hablaba en 1963 de inventar el futuro. En eso estamos y en eso deben estar. Más bonito todavía, el cardiólogo Bernard Lawn, fundador de la Asociación Internacional de Médicos para la Prevención de la Guerra Nuclear y premio Nobel de la Paz en 1985, decía que 'sólo aquel que ve lo invisible es capaz de hacer lo imposible’. Ese es su reto.
Y ese es el camino ilusionante que inician hoy y para el que les deseo, a título personal y en nombre de esta facultad que hoy los acoge, toda suerte de venturas.

Bibliografía

El País, 24 de julio de 2011. p. 31

2. Bardes CL. Defining 'patient-centered medicine'. N Engl J Med 2012; 366: 782-3.

3. Truog RD. Patients and doctors -the evolution of a relationship. N Engl J Med 2012; 366: 581-5.

4. NEJM@200 -two centuries at the Journal. N Engl J Med 2012; 366: 83.

5. Stepan NL. Eradication: ridding the world of diseases forever? London: Reaktion Books; 2011.

6. Brandt AM. A reader's guide to 200 years of the New England Journal of Medicine. N Engl J Med 2012; 366: 1-7.

7. Editorial. Practice of medicine for married women. N Engl J Med 1955; 25O: 486

8. Reuben DB, Tinetti ME. Goal-oriented patient care -an alternative health outcome paradigm. N Engl J Med 2012; 366: 777-9. 